\title{
Klassisches Kaposi-Sarkom mit ausgedehnter Hautbeteiligung
}

\author{
H. Friedlein ${ }^{1}$ \\ M. Mittag ${ }^{1}$ \\ M. Rytter ${ }^{1}$ \\ U. Gütz ${ }^{2}$ \\ U.-F. Haustein ${ }^{1}$
}

\author{
Classical Kaposi's Sarcoma with Extensive Skin Lesions
}

\section{Zusammenfassung}

Das klassische, nicht HIV-assoziierte Kaposi-Sarkom ist primär ein Hauttumor, der vor allem die untere Extremität älterer Männer mediterraner Abstammung betrifft. Am Beispiel eines 77-jährigen Mannes zeigen wir eine ausgeprägte Krankheitsmanifestation und beschreiben die epidemiologischen und klinischen Merkmale der Erkrankung. Die Therapie des Kaposi-Sarkoms ist in der Regel palliativ bei allerdings langsamem Fortschreiten der Krankheit. Wir stellen lokale und systemische Behandlungsmöglichkeiten vor. Anamnese: 77-jähriger Mann mit der Erstdiagnose eines klassischen Kaposisarkoms 1992. Vorbehandlung mit Prospidin. Aufnahmebefund: Ausgedehnte subkutan-kutan lokalisierte livide-rötliche Gefäßtumoren v.a. an der gesamten unteren Extremität und unteren Stamm. Diagnostik: HHV-8-Nachweis in Blut und Tumorgewebe; keine Beteiligung innerer Organe detektierbar. Therapie und Verlauf: Ablehnende Haltung des Patienten gegenüber vorgeschlagenen Therapieoptionen. Diskussion/Folgerung: Es wird eine multifaktorielle Ätiologie angenommen. Eine starke Assoziation mit dem HHV-8 ist nachgewiesen. Es gibt verschiedene lokale und systemische Therapieansätze, aber keine Standardtherapie für das klassische Kaposi-Sarkom. Die Therapienebenwirkungen sind gegen die -erfolge bei oft sehr langsamer Tumorprogression abzuwägen.

\section{Abstract}

Classical HIV-negative Kaposi's sarcoma is primarily a skin disease of the lower extremities affecting predominantly elderly men of Mediterranean origin. We report a case of a 77 year old man with an extensive manifestation of disease, and we describe the clinical and epidemiological characteristics. Therapy of classical Kaposi's sarcoma is always palliative, however, there is slow progress of disease. We present ways of local and systemical treatment. History: A 77 year old patient being diagnosed for classical Kaposi's sarcoma in 1992. Previous treatment with Prospidine. Physical Examination: Extensive subcutanous-cutanous localized livid vascular tumors which predominantly infiltrate the lower extremities and lower trunk. Diagnostics: Paraclinic not remarkable. Human Herpes-Virus-8 is detectable both in tumor tissue and blood. Therapy/progress: Patient did not wish any treatment. Discussion: Etiology is still unclear. A strong association with HHV-8-infection has been established. There is no standard treatment for CKS, so various possibilities of topical and systemic treatment have been described. Side-effects and benefits of any treatment are to be evaluated for it's a slow growing tumor.
1872 beschrieb Moritz Kaposi bei älteren Männern jüdischer Abstammung ein idiopathisches multiples Pigmentsarkom. War es in Nordeuropa bis in die 60er Jahre eine Seltenheit, so sieht man das Kaposi-Sarkom (KS) heute deutlich häufiger in Verbindung mit AIDS und Immunsuppression.
Das Kaposi-Sarkom stellt einen multifokalen Gefäßtumor dar, der sich vorwiegend in der Haut mit rötlich-lividen bis braunschwarzen Maculae, Papeln, Noduli und Plaques manifestiert. Auch ein Befall der inneren Organen (bei ca. 10\%) ist möglich.

Histologisch finden sich in frühen Läsionen bizarr geformte, teils abortive Gefäßschlitze und Extravasate von Erythrozyten. Später

${ }^{1}$ Universitätshautklinik Leipzig (Direktor: Prof. Dr. U.-F. Haustein)

${ }^{2}$ Institut für Pathologie der Universität Leipzig (Direktor: Prof. Dr. C. Wittekind) 


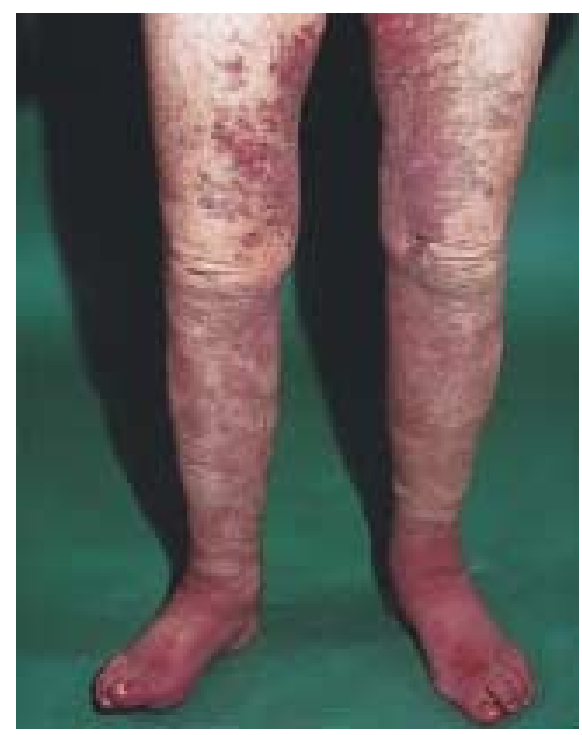

Abb. 1 Ausgedehnte, flächige, livid-rötliche Gefäßtumoren an der unteren Extremität.

überwiegen dicht gepackte Aggregate spindeliger Tumorzellen, die vermutlich von lymphatischen Endothelzellen abstammen.

Üblicherweise wird die Erkrankung in vier Subtypen eingeteilt, die Verschiedenheiten in der Epidemiologie, Prognose und im klinischen Verlauf erwarten lassen: 1) das klassische KS, 2) das endemische zentralafrikanische $\mathrm{KS}, 3$ ) das iatrogene, immunsuppressions- und medikamentenassoziierte KS und schließlich 4) das HIV-assoziierte KS.

Die letzteren drei Untergruppen kennzeichnet ein aggressiver Krankheitsverlauf. Histologisch lassen sich die vier Typen jedoch nicht unterscheiden.

Das klassische KS findet man vor allem bei Männern über 60 Jahre, die im Mittelmeerraum oder in Osteuropa leben bzw. von dort stammen. Auffallend ist der hohe Anteil von Juden. Allgemein sind die Gefäßtumoren besonders an den unteren Extremitäten lokalisiert. Einzelfallbeschreibungen bei jungen, gesunden und HIV-negativen Erwachsenen liegen vor $[3,9]$.

\section{Kasuistik}

\section{Anamnese}

Wir berichten über einen 77 Jahre alten Mann jüdischer Abstammung aus Litauen. 1992 bemerkte er das Auftreten von lividerötlichen Hauterscheinungen an beiden Unterschenkeln. Drei Jahre später wurde die Diagnose eines klassischen Kaposi-Sarkoms histologisch im Republikanischen Onkologischen Klinikum Riga gestellt. Seither hatte der Patient mehrere Zyklen Chemotherapie erhalten, zuletzt im Oktober 1999. Verwendet wurde das Präparat Prospidinum lyophilisatum in einer Gesamtdosis von 4,0 g. Darunter wurde nur eine partielle Tumorregression beobachtet. Bei der Vorstellung in unserer Klinik wurden vor allem Klagen über plantar lokalisierte Schmerzen aufgrund einer ausgedehnten Tumorausbreitung vorgebracht. An Nebendiagnosen lagen vor: nicht insulinpflichtiger Diabetes mellitus Typ II, koronare Herzkrankheit Stadium II (nach Einteilung der New York Heart Association) und eine allgemeine Arteriosklerose so- wie ein Zustand nach Nephrektomie links und Prostatektomie (beides 1986).

\section{Erst-/Aufnahmebefund}

77-jähriger, leicht adipöser Patient in deutlich reduziertem Allgemeinzustand. Blutdruck 120/70 mm Hg, Puls 72/min., inguinal beidseits bohnenkerngroße Lymphknoten tastbar; Fußpulse nicht palpabel, sonstige orientierende internistische Untersuchung unauffällig.

\section{Hautbefund}

An der unteren Extremität fanden sich distal ausgedehnte flächig-konfluierende, proximal hingegen eher einzelstehende livide-rötliche, sowohl infiltrativ plaqueartige als auch makulöse, vorwiegend kutan-subkutan lokalisierte Gefäßtumoren. Zum Teil waren sie entlang der Hautspaltlinien angeordnet (Abb.1 und 2). Beide Füße waren lila-livide verfärbt und kalt. Rechts plantar wurde ein deutlicher Druckschmerz angegeben.

Auch an der Bauchwand fanden sich zwei solitäre, rötliche ca. $1,5 \mathrm{~cm}$ große Infiltrate. Ähnliche Veränderungen ließen sich auch beugeseitig am linken Unterarm und am rechten Handrücken nachweisen. Allerdings war ihr Durchmesser mit ca. $6 \mathrm{~cm}$ bzw. $5 \mathrm{~cm}$ deutlich größer. Das übrige Integument ist erscheinungsfrei.

\section{Befunde diagnostischer Untersuchungen Labordiagnostik}

Routinelaborparameter bis auf eine leichte Leukozytose von $12,1 \times 10^{9} / 1$ normwertig.

\section{Serologie HIV 1 und 2}

Negativ. Untersuchung der Lymphozytensubpopulation:

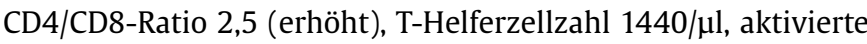
T-Zellen $12,8 \%$.

\section{Serologie Humanes Herpes Virus-8}

Seropositivität im Anti-HHV 8 IgG-ELISA, Nichtvorhandensein von HHV 8-DNA in peripheren Blutzellen bei gleichzeitigem Nachweis von HHV 8-DNA im Tumorgewebe.

\section{Apparative Diagnostik}

Thorax-Röntgenaufnahme, Ultraschalluntersuchung des Abdomens und der Lymphknoten, Rekto- und Ösophago-Gastro-Duodenoskopie: kein Nachweis von Kaposi-Sarkomen in inneren Organen.

\section{Histologische Untersuchung}

Von Hautbioptat aus Tumor am rechten Oberschenkel (1041/00 Universitätshautklinik Leipzig, H 05794 - 00 Pathologisches Institut Universität Leipzig): Exzisat mit atropher Epidermis. Die oberen und mittleren Anteile des Stratum reticulare und des Bindegewebes werden durchsetzt von fokal gezackt erscheinenden und die kollagenen Fasern dissezierenden vaskulären Proliferaten, die locker und unregelmäßig von Kollagenbündeln umgeben sind. Perivaskulär lockere lymphozytäre und teils auch plasmazelluläre Infiltration. Ab und an findet man dilatierte lymphgefäßähnliche Räume; Adnexstrukturen werden von den neoplastischen Gefäßen umgriffen. Es ist eine Ablagerung von hyalinen Globuli in der PAS-Färbung sowie von Hämosiderin nachweisbar. 


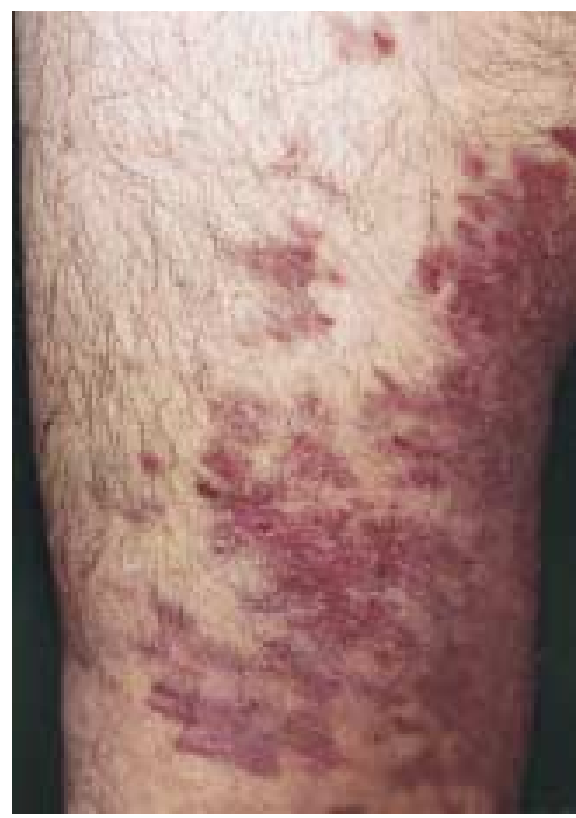

Abb. 2 Teils plaqueartige, teils makulöse Läsionen entlang der Hautspaltlinien.

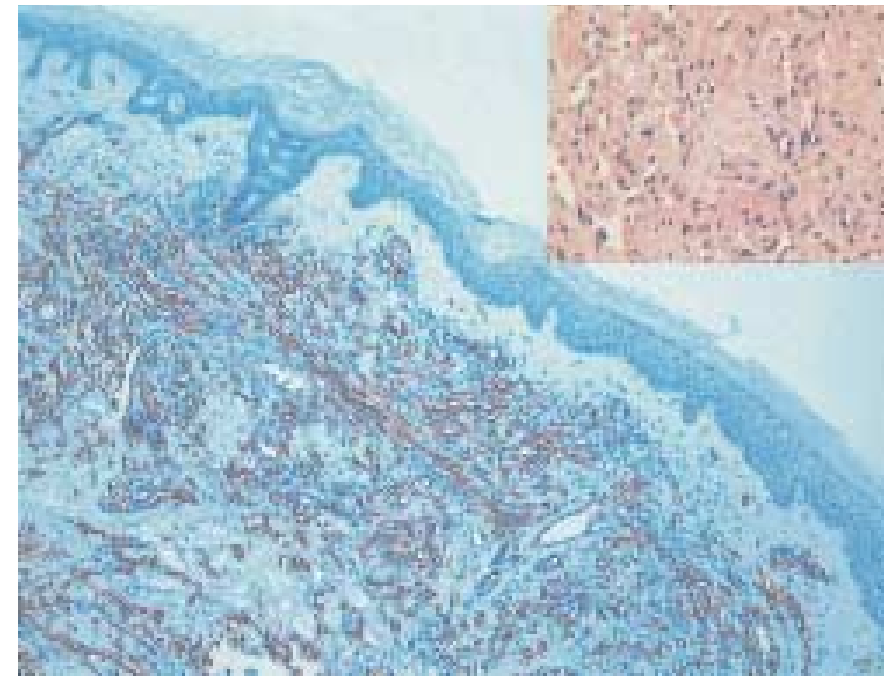

Abb. 3 Dermales Proliferat atypischer kapillärer Gefäße (CD 34, Objektiv $10 \times$ ). Inset oben rechts: irreguläre kapilläre Gefäßspalten, die von mäßig polymorphen, hyperchromatischen Endothelien ausgekleidet werden $(\mathrm{HE}, 63 \times)$.

Die Immunreaktion mit CD 34 ergibt eine stark positive Reaktion der neoplastischen und der originären Gefäße.

\section{Beurteilung}

Klassisches Kaposi-Sarkom vom Patch- und Plaquestadium (Abb. 3).

\section{Therapie und Verlauf}

Bei ausschließlich kutaner Tumorausbreitung schlugen wir dem Patienten in Zusammenarbeit mit der Strahlentherapeutischen Abteilung der Universität Leipzig eine großflächige lokale radiologische Behandlung mit schnellen Elektronen (geplante Gesamtdosis $30 \mathrm{~Gy}$ ) vor. Alternativ wurden eine systemische Therapie mit Vinblastin, Low-dose-Interferon oder liposomalem Doxorubicin $\left(\right.$ Caelyx $\left.^{\circledR}\right)$ diskutiert.
Nach erbetener Bedenkzeit über das weitere Prozedere entzog sich der Patient bisher jeglicher Therapie.

\section{Diskussion}

Die Pathogenese des KS wird trotz neuer Forschungsergebnisse kontrovers diskutiert: Es werden genetische Disposition, Umwelteinflüsse und Infektionen hier berücksichtigt.

Die genetischen Faktoren umfassen die ethnische Zugehörigkeit und das Vorliegen bestimmter Histokompatibilitätsantigene, vor allem HLA-DR 5 [12]; wir haben unseren Patienten nicht daraufhin untersucht. Typisch ist jedoch die Herkunft vieler Patienten (jüdische Abstammung, Osteuropa). Diese kann aufgrund niedriger Hygienestandards ein erhöhtes Risiko für Infektionen und damit Immundysregulationen [5] bedingen.

1994 identifizierten Chang et al. [13] herpesvirusähnliche DNASequenzen in den KS-Läsionen HIV-positiver Patienten. Inzwischen wird dieses Virus als Herpesvirus Typ 8 (HHV 8) oder KSassoziiertes Herpesvirus (KSHV) bezeichnet. HHV 8 scheint im Gegensatz zu den anderen humanen Herpesviren nicht ubiquitär vorzukommen. Auffallend sind starke regionale Unterschiede [10].

Obwohl bisher kein Beweis erbracht werden konnte, spricht doch die sehr starke Assoziation dieses Virus mit dem KS für dessen ursächliche Bedeutung bei der Tumorgenese. In einer italienischen Studie [11] wurde HHV 8-DNA bei allen KS-Patienten $(\mathrm{n}=33)$ intra- und periläsional, aber nur bei $33 \%$ in gesunder Haut detektiert. Im Blut waren in 69,6\% DNA-Sequenzen nachweisbar im Gegensatz zu 23,8\% in der gesunden Kontrollgruppe. In letzterer konnte in der Haut sogar überhaupt kein HHV 8 gefunden werden.

Auch bei unserem Patienten ließen sich im Blut anti-HHV 8-IgG Antikörper identifizieren und intraläsional, d.h. im Tumorgewebe, Virus-DNA nachweisen.

Bis heute gibt es keine Heilung für Patienten mit klassischem Kaposi-Sarkom. Alle Therapien sind palliativ und zeigen nur temporäre Effekte.

Beim klassischen KS wird eine fraktionierte Radiatio von insgesamt 10 bis $40 \mathrm{~Gy}$ Gesamtdosis z. B. mit weicher Photonenbestrahlung oder Röntgenstrahlen favorisiert. Sie vermag das Tumorwachstum zu verlangsamen oder auf Zeit zu stoppen. Bei großflächigem Befund ist die Therapie allerdings aus physikalischen Gründen nur schwer durchzuführen (großer Abstand Strahlenquelle - Patient). Therapiebedingte Blutbildungsstörungen sind beim alten Menschen nicht zu erwarten, da die Hämatopoese in deren Röhrenknochen nur noch minimal ist. Ansprechraten bis zu $92 \%$ wurden beschrieben $[1,5,13]$, zu einer Beschwerdelinderung kam es bei allen Patienten [1].

Die Kryotherapie bietet eine komplikationsarme und einfache Behandlungsoption für kleinere Herde. Einzeltumoren können auch operativ oder mit dem Laser (z. B. Nd:YAG-Laser oder Farbstofflaser) entfernt werden. 
In Einzelfällen wurden die Läsionen erfolgreich mit Virustatika wie Cidofovir (VISTIDE ${ }^{\circledR}$ ) oder Zytostatika wie Vinblastin (Cellblastin $^{\circledR}$ ) [3] unterspritzt.

Systemisch gibt es für das klassische KS keine Standardbehandlung. Das Ansprechen auf eine Chemotherapie ist von Fall zu Fall unterschiedlich. Bei dem zumeist verwendeten Vinblastin wurden Erfolgsraten von bis zu 90\% angegeben [13,14]. Zidan et al. verabreichten $6 \mathrm{mg}$ Vinblastin alle zwei Wochen i.v. bei tolerablen Nebenwirkungen [16]. Es werden auch Kombinationsschemata wie CHOP, ABVD oder MOPP versucht.

Tur und Brenner [7] berichteten über die erfolgreiche Therapie mit Low-dose-Interferon Alpha beim ausgedehnten KS (3-mal 2-3 MIU s.c. wöchentlich). Wichtig erscheint, dass im Gegensatz zum HIV-assoziierten KS keine kontinuierliche Behandlung notwendig ist. Rezidive sprachen auf einer Wiederaufnahme der Behandlung stets gut an. Allerdings muss für eine effektive Therapie die Krankheit offenbar aktiv, d. h. progredient sein.

Vielversprechend scheint auch liposomal enkapsuliertes Doxorubicin $\left(\right.$ Caely ${ }^{\circledR}$ ) besonders wegen der dadurch erreichten besseren Verträglichkeit.

Grundsätzlich sind beim klassischen Kaposisarkom die Nebenwirkungen einer systemischen Chemotherapie gegen den langsamen, die Lebenszeit meist nicht limitierenden Verlauf der Erkrankung abzuwägen. Brenner et al. [13] halten bei voller Immunkompentenz des Patienten die Beobachtung für ausreichend.

\section{Literatur}

${ }^{1}$ Tombolini V, Osti MF. Radiotherapy in CKS: experience of the Institute of Radiologiy of University „ La Sapienzy“ of Rome. Anticancer Res 1999; 19: $4539-4544$

2 Iscovich J, Boffetta P. CKS: epidemiology and risk factors. Cancer 2000; 88: $500-517$

${ }^{3}$ Erdem T, Atasoy M. A juvenile Case of CKS. Acta Derm Venerol 1999; 79: $492-493$

${ }^{4}$ Touloumi G, Hatzakis A. The role of immunosuppression and immune-activation in CKS. Int J Cancer 1999; 82: 817-821

${ }^{5}$ Fenig E, Brenner B. CKS: experience at Rabin Medical Center in Israel. Am J Clin Oncol 1998; 21: 498-500

${ }^{6}$ Pfrommer C, Tebbe B. Progressive HHV-8-positive CKS: rapid response to interferon alpha-2a but persistence of HHV-8 DNA sequences in lesional skin. Br J Dermatol 1998; 139: 516-519

7 Tur E, Brenner S. CKS: low-dose interferon alfa treatment. Dermatology 1998; 197: 37-42

8 Iscovich J, Boffetta P. CKS in Arabs living in Israel, 1970 - 1993: a population-based incidence study. Int J Cancer 1998; 77: 319-321

${ }^{9}$ Potouridou I, Katsambas A. CKS in two heterosexual men. J Europ Acad Derm Venerol 1998; 10: 48-52

10 Tschachler E. Das humane Herpesvirus Typ 8. Hautarzt 2000; 51: $623-629$

${ }^{11}$ Cattani P, Capuano M. HHV 8 in Italian HIV-Seronegative Patients with KS. Arch. Dermatol 1998; 134: 695-699

12 Pollach MS, Safai B. Frequencies of HLA and Gm immunogenetic markers in KS. Tissue Antigens 1983; 21: 1-8

${ }^{13}$ Chang Y, Cesarman E. Identification of herpesvirus-like DANN sequences in AIDS-associated KS. Science 1994; 266: 1865-1869

${ }^{14}$ Brenner B, Rakoxsky E. Tailoring Treatment for CKS. Comprehensive Clinical Guidelines. Int J Oncol 1999; 14: 1097-1102

${ }^{15}$ Richman SP, Vogel CL. Kaposi's sarcoma. In: Brain MC, Carbone PP (eds). Current Therapy in Hematology-Oncology. Current Therapy Series. Toronto: Decker, 1988; 3: 353-355

16 Zidan J, Robenstein W, Abzah A, Taman S. Treatment of Kaposi's sarcoma with vinblastine in patients with disseminated disease. Isr Med Assoc J 2001; 3: $251-253$ 